\title{
Diffusion tensor imaging of the roots of the brachial plexus: a systematic review and meta-analysis of normative values
}

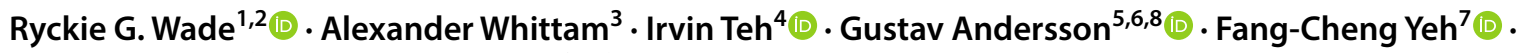 \\ Mikael Wiberg ${ }^{5,6}$ (D) Grainne Bourke $\mathrm{G}^{1,2,5,6}$ (1)
}

Received: 19 May 2020 / Accepted: 25 September 2020 / Published online: 9 October 2020

(c) The Author(s) 2020

\begin{abstract}
Purpose Diffusion tensor magnetic resonance imaging (DTI) characterises tissue microstructure and provides proxy measures of myelination, axon diameter, fibre density and organisation. This may be valuable in the assessment of the roots of the brachial plexus in health and disease. Therefore, there is a need to define the normal DTI values.

Methods The literature was systematically searched for studies of asymptomatic adults who underwent DTI of the brachial plexus. Participant characteristics, scanning protocols, and measurements of the fractional anisotropy (FA) and mean diffusivity (MD) of each spinal root were extracted by two independent review authors. Generalised linear modelling was used to estimate the effect of experimental conditions on the FA and MD. Meta-analysis of root-level estimates was performed using Cohen's method with random effects.

Results Nine articles, describing 316 adults (1:1 male:female) of mean age 35 years (SD 6) were included. Increments of ten diffusion sensitising gradient directions reduced the mean FA by 0.01 (95\% CI 0.01, 0.03). Each year of life reduced the mean MD by $0.03 \times 10^{-3} \mathrm{~mm}^{2} / \mathrm{s}(95 \%$ CI $0.01,0.04)$. At 3 -T, the pooled mean FA of the roots was 0.36 (95\% CI $0.34,0.38$; $\left.I^{2} 98 \%\right)$. The pooled mean MD of the roots was $1.51 \times 10^{-3} \mathrm{~mm}^{2} / \mathrm{s}$ (95\% CI $\left.1.45,1.56 ; I^{2} 99 \%\right)$.

Conclusions The FA and MD of the roots of the brachial plexus vary according to experimental conditions and participant factors. We provide summary estimates of the normative values in different conditions which may be valuable to researchers and clinicians alike.
\end{abstract}

Keywords Diffusion tensor $\cdot$ Brachial plexus $\cdot$ Normal $\cdot$ Normative $\cdot$ Healthy $\cdot$ Peripheral nerve

Electronic supplementary material The online version of this article (https://doi.org/10.1007/s40336-020-00393-x) contains supplementary material, which is available to authorized users.

Ryckie G. Wade

ryckiewade@gmail.com

1 Department of Plastic and Reconstructive Surgery, Leeds Teaching Hospitals Trust, Leeds LS1 3EX, UK

2 Leeds Institute for Medical Research, University of Leeds, Leeds, UK

3 Sheffield Teaching Hospitals NHS Foundation Trust, Sheffield, UK

4 Leeds Institute for Cardiovascular and Metabolic Medicine, University of Leeds, Leeds, UK

\section{Introduction}

The brachial plexus is a network of nerves which supply the upper limb with movement and feeling (Fig. 1 and Supplementary Fig. 1). Magnetic resonance imaging (MRI) is generally considered the best non-invasive imaging modality for

5 Department of Integrative Medical Biology, Faculty of Medicine, Umeå University, Umeå, Sweden

6 Department of Surgical and Perioperative Science, Section for Hand and Plastic Surgery, Umeå University, Umeå, Sweden

7 Department of Neurological Surgery, University of Pittsburgh, Pittsburgh, USA

8 Wallenberg Centre for Molecular Medicine, Umeå University, Umeå, Sweden 
Fig. 1 The roots of the brachial plexus emerging from the intervertebral foramina (upper left image) and their relationship to the scalene muscles and vasculature of the upper limb (upper right image). The lower image is a simplified schematic of the brachial plexus highlighting (in purple) the spinal roots. Reproduced with permission from Mr. Donald Sammut



diagnosing various pathologies affecting the brachial plexus [1-6]. The roots of the brachial plexus are the most common site of injury [7] and typically, the status of the root dictates the prognosis and surgical reconstruction. Consequently, defining the health status of the roots is of paramount importance. Whilst MRI is more accurate than electrophysiology $[2,6]$, ultrasonography $[3-5,8]$ and computed tomography myelography [9], the diagnostic performance of conventional cross-sectional MRI for assessing the spinal nerve roots remains suboptimal [7]. Consequently, there has been a surge of research into diffusion tensor imaging (DTI) which may provide additional valuable information. DTI characterises tissue microstructure and provides reproducible [10-13] proxy measures of nerve health which are sensitive to myelination, axon diameter, fibre density and organisation $[14,15]$. The parameters typically derived from DTI include the fractional anisotropy (FA), mean diffusivity (MD), axial diffusivity (AD) and radial diffusivity (RD). FA is a scalar value between zero and one; an FA of zero implies isotropic diffusion within a voxel, whilst (in the context of peripheral nerve imaging) a FA nearing one implies diffusion along a single axis i.e., either anterograde of retrograde diffusion along the length of the nerve. MD describes the average molecular diffusion rate of the tensor; $\mathrm{AD}$ describes the diffusion rate in the long axis and $\mathrm{RD}$ represents diffusion perpendicular to the long axis.

Before researchers and clinicians can use DTI parameters to identify abnormal roots of the brachial plexus, there is a need to define the "normal" values. Numerous studies have examined aspects of DTI of the brachial plexus in healthy adults. This review aims to summarise the values observed in health and explore discrepancies in the reported measurements.

\section{Methods}

This review is registered with PROPSERO (ID CRD42019155788), it was designed and conducted in accordance with the Cochrane Handbook of Systematic 
Reviews [16] and has been authored in accordance with the PRISMA checklist [17].

\section{Types of studies}

We included all studies which reported the findings of diffusion tensor magnetic resonance imaging of the roots of the brachial plexus in healthy adults. Case reports were excluded.

\section{Participants}

Asymptomatic adults (aged $\geq 16$ years) with no known pathology (past or present) affecting the spinal cord or brachial plexus were the population of interest.

\section{Image acquisition}

Studies must have reported diffusion tensor imaging parameters from the roots of the brachial plexus.

\section{Search strategy}

The NICE Healthcare Databases (hdas.nice.org.uk) was searched using the terms "diffusion tensor" OR "DTI" AND "brachial plexus". This yielded 67 hits in PubMed, 36 in Embase, 8 in CINAHL, 2 in CENTRAL and 2 in ClinicalTrials.gov the on 13th November 2019. After de-duplication, 78 unique citations were independently screened by two review authors (RGW and AW). The full texts of all potentially relevant articles were obtained. The reference lists for included articles were also scrutinised for potentially relevant papers. The final lists of included articles were compared and disagreements resolved by discussion.

\section{Study selection}

Two review authors (RGW and AW) independently screened titles and abstracts for relevance, in accordance with the eligibility criteria. The full texts of potentially eligible articles were obtained and again independently assessed by the same two authors. Disagreements were resolved by discussion.

\section{Data extraction}

Two review authors (RGW and AW) independently extracted data concerning the demographics, scanner, pulse sequence, pre-processing, tensorial reconstruction, measurement conditions and the outcomes of interest. The spinal nerve root was the unit of analysis [7] and root-level estimates of DTI parameters were extracted. Where data was missing or unclear, the corresponding author was contacted by email and/or phone and if no reply was received, 4 weeks later all authors were contacted in addition to re-contacting the corresponding author. The authors of one study [18] provided additional information (measurements form the extraforaminal roots using identical methods) for the purposes of this review.

\section{Outcomes}

The primary outcome is to estimate the normal fractional anisotropy (FA) of the extraforaminal roots of the brachial plexus in healthy adults. The secondary outcomes include: (a) to estimate the normal mean diffusivity (MD) of the extraforaminal roots of the brachial plexus in healthy adults, and (b) to explore the associations between DTI parameters and participants age, the signal-to-noise (SNR) ratios and related factors such as the $b$ value(s), echo time(s) (TE) and resolution (in cubic millimetres, $\mathrm{mm}^{3}$ ) and the number of diffusion sensitising gradient directions $\left(\mathrm{N}_{\mathrm{D}}\right)$ sampled per shell.

\section{Methodological quality assessment}

The risk of bias was assessed by two review authors (RGW and AW) using the ROBINS-I tool [19] and displayed graphically using robvis [20]. Disagreements were resolved by discussion.

\section{Statistical analysis}

Data were analysed in Stata/MP v15 (StataCop LLC, Texas). To estimate the effect of experimental/participant factors on the FA and MD, generalised linear modelling (GLM) was used with gaussian families. As the TE, $b$ value and resolution are functions of SNR, the fixed effects were selected to be SNR, age in years and the $N_{\mathrm{D}}$ all of which were handled as continuous variables. The random effects in the GLM varied by the study. Estimates were bootstrapped using lossless non-parametric resampling with replacement, with 1000 iterations. There was insufficient data to meaningfully assess the effect of different tensor fitting methods or components of the $b$ value (diffusion time, magnitude or interval) on DTI parameters. To visualise the association of FA with $N_{\mathrm{D}}$, and MD with age, scatterplots of the aggregate estimates were generated using the metareg package; the circles are root-level estimates and the sizes are dependent on the precision (inverse variance) of the estimate. To estimate the pooled normal FA and MD of the spinal roots, meta-analyses were performed using the metan package. Cohen's method was used because both FA and MD were homoscedastic. Dersimonian and Laird random effects were used given the clinical heterogeneity. 
Analyses were subgrouped by both the $\mathrm{N}_{\mathrm{D}}$ and spinal root (C5, C6, C7, C8, T1). Confidence intervals (CI) were generated to the $95 \%$ level. To assess the possibility of small-study effects we constructed a funnel plot using the metafunnel package, which is a scatterplot of the effect size against precision; symmetry implies the absence of small-study effects.

\section{Results}

After reviewing 27 full texts, 15 were excluded (Supplementary materials) and 9 articles (of 9 unique studies) were included [18, 21-28] (Fig. 2).

\section{Study characteristics}

Overall, 316 adults were included. The ratio of males:females was approximately 1:1 (156:154). The mean age of participants was 35 years (SD 6). The characteristics of included studies are shown in Table 1.

\section{Risk of bias within studies}

The risk of bias for the included studies are summarised in Fig. 3. Eight of the included studies [18, 21-24, 26, 28, 29] were at risk of bias due to confounding because there was no adjustment for a) effect modifiers such as age, weight and the experimental factors described above, and b) repeated measures, i.e. ten estimates of the FA (one from each spinal root, bilaterally) taken from the same individual will be highly correlated and without adjustment, the sample-level

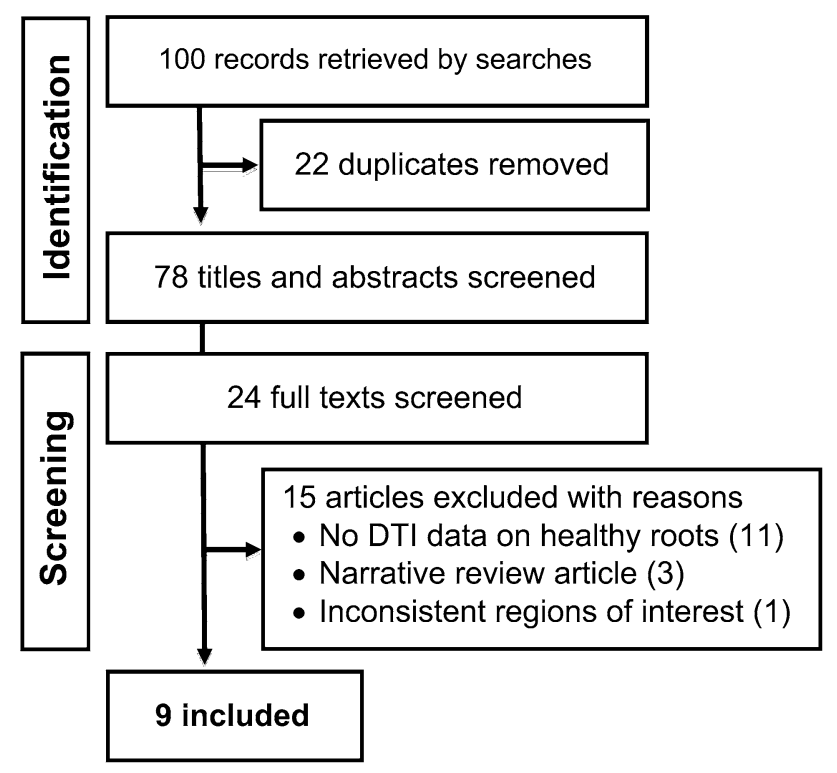

Fig. 2 PRISMA flowchart estimates will have falsely small variances. Seven studies [21-25, 28] provided no information about missing data and so the risk of bias is unclear. Two studies [21,23] were judged to be at high risk of bias in the measurement of FA and MD because a bespoke region of interest was used to calculate the FA and the number of diffusion sensitising gradients used was not described [21]. Three studies [24, $25,28]$ were at unclear risk of bias in the measurement of FA and MD because information was lacking about preprocessing, tensorial reconstruction or how the estimates of the FA and MD were derived from images (e.g. region of interest size and position). One study [23] was at high risk of reporting bias because three $b$ values were tested $(700,900$, $1100 \mathrm{~mm} / \mathrm{s}$ ) but it is unclear which yielded the estimates of FA and MD reported in the manuscript or whether they are an average of the three. The risk of bias due to selective outcome reporting was unclear in six studies [21-24, 26, 28], because no information was provided regarding the exclusion criteria, attrition due to scan intolerance, dataset exclusion (e.g. for uncorrectable motion artefact) or otherwise and there were no published protocols to consult.

\section{Evidence synthesis}

There were no clear associations between experimental factors and the FA or MD on univariable modelling (Table 2). Multivariable modelling showed that the angular resolution was strongly associated with FA, whereby every additional ten diffusion sensitising gradient directions sampled reduced the FA by 0.01 (95\% CI 0.01, 0.03; Fig. 4). Furthermore, multivariable modelling showed that each year of life reduced the MD by $0.03 \times 10^{-3} \mathrm{~mm}^{2} / \mathrm{s}(95 \%$ CI $0.01,0.04$; Fig. 5). Bootstrapping did not change these estimates.

Seven studies [18, 21, 22, 24, 27-29] were included in the meta-analysis of the normal FA of the roots of the brachial plexus at $3 \mathrm{~T}$; one study [26] did not provide estimates of the variance so could not be included. The pooled estimate of the normal FA of the root was 0.36 (95\% CI 0.34, 0.38; Fig. 6). There were no statistically significant differences between the five roots. However, there was significant statistical heterogeneity between studies $\left(I^{2} 98 \%\right)$ which may be related to the experimental conditions described above. The pooled estimates of the normal FA subgrouped by spinal root (including the study performed at $1.5 \mathrm{~T}$ which had usable data [23]) are shown in Supplementary Fig. 2.

Six studies [18, 22, 24, 27-29] contributed to the metaanalysis of the normal MD of the roots of the brachial plexus at $3 \mathrm{~T}$; one study [26] did not provide estimates of the variance and one study [21] did not report the MD of any roots. The pooled estimate of the normal MD of the roots of the brachial plexus was $1.51 \times 10^{-3} \mathrm{~mm}^{2} / \mathrm{s}(95 \%$ CI $1.45,1.56$; Fig. 7 and Supplementary Fig. 3); however, there was high statistical heterogeneity between studies $\left(I^{2} 99 \%\right)$ which may 


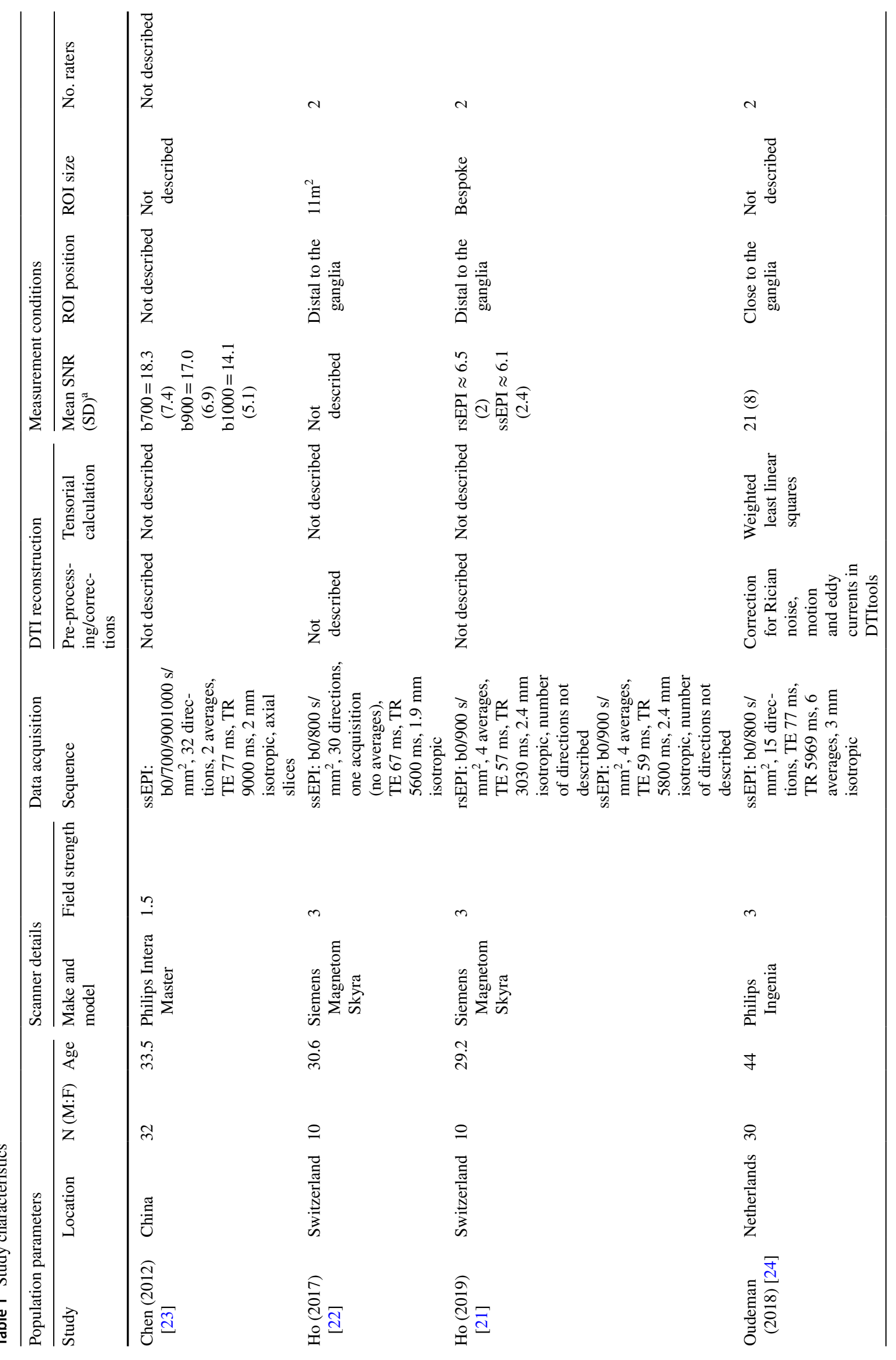




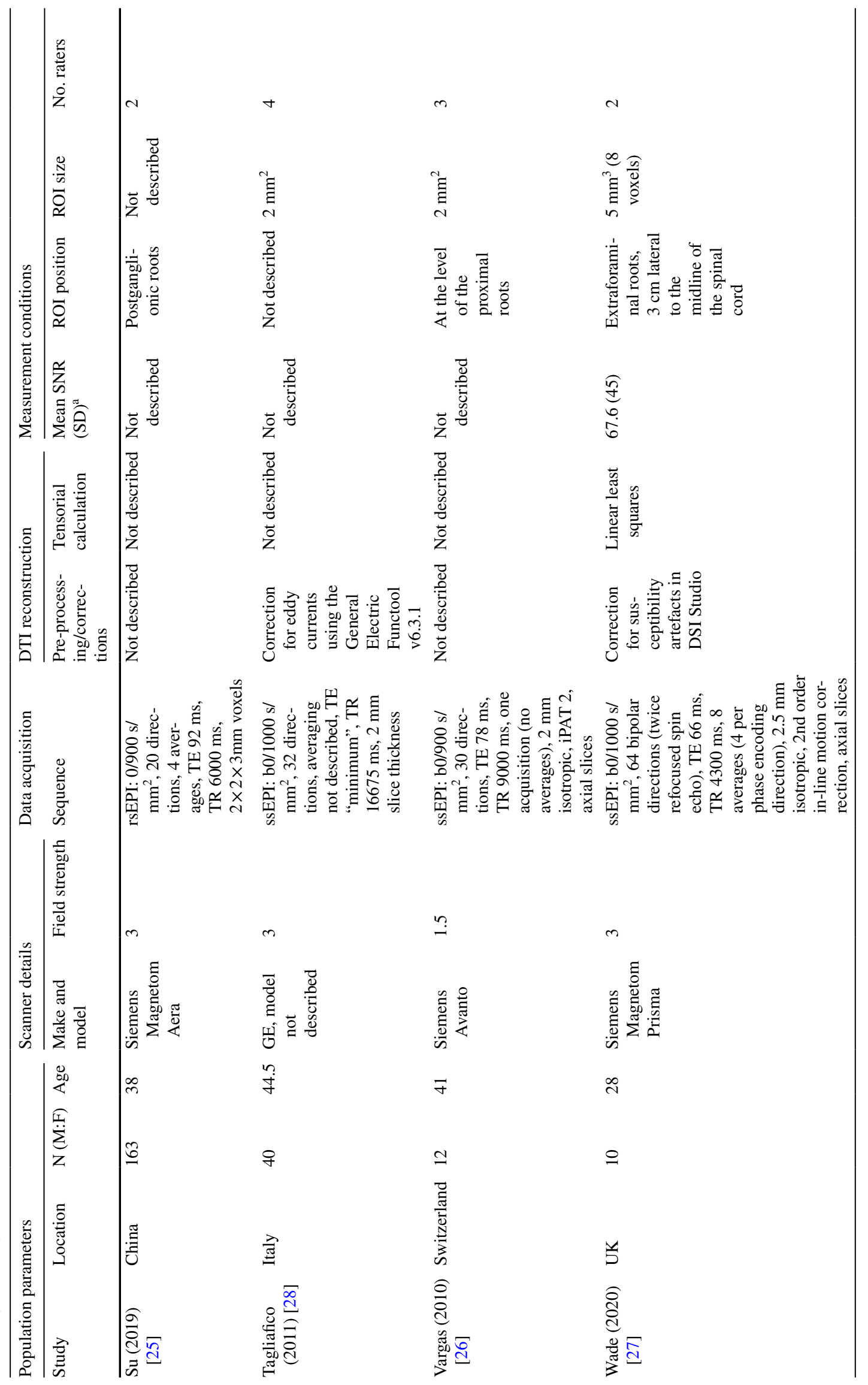




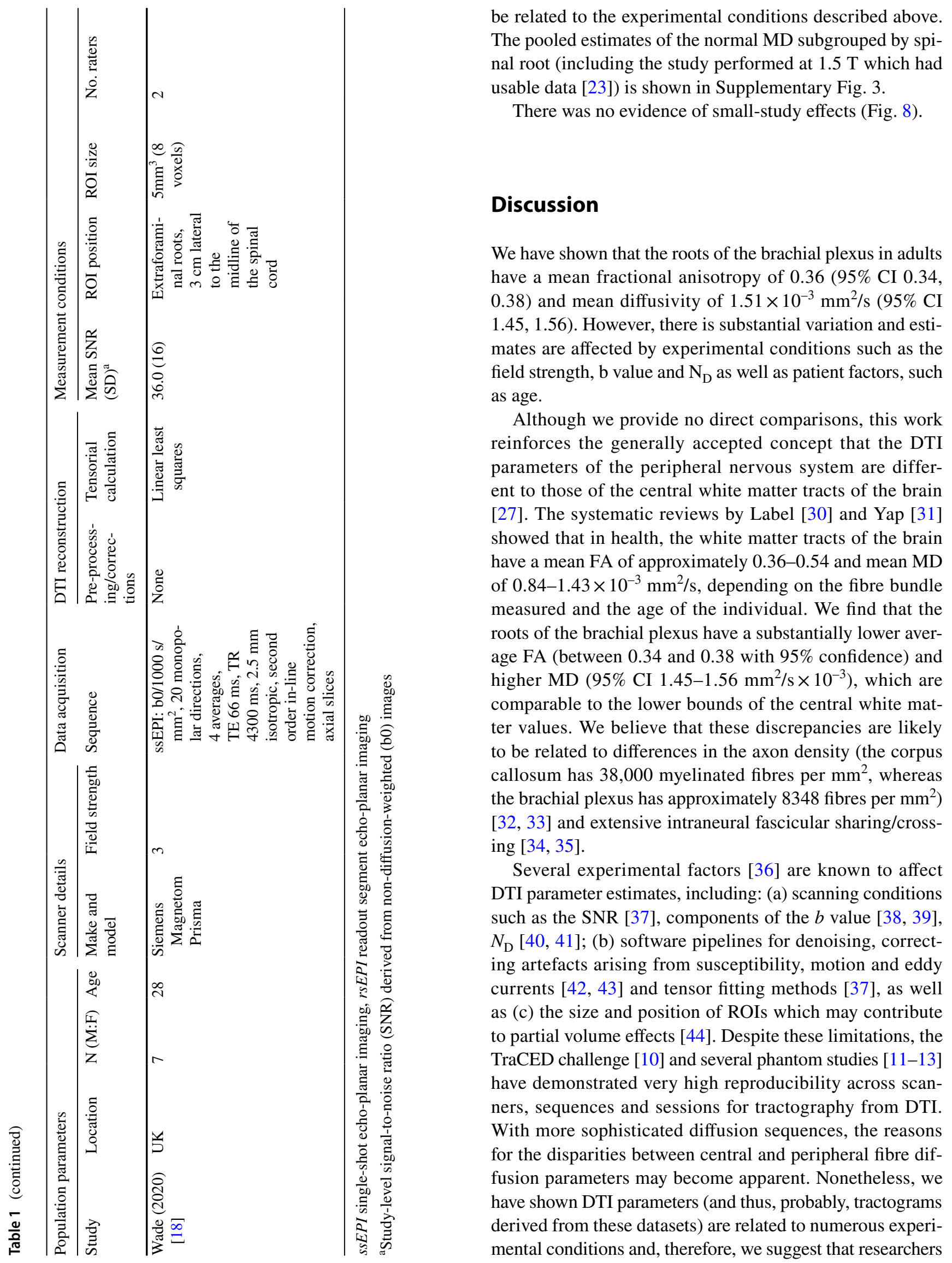




\begin{tabular}{|c|c|c|c|c|c|c|c|c|}
\hline & D1 & $\mathrm{D} 2$ & D3 & D4 & D5 & D6 & D7 & Overall \\
\hline \multicolumn{9}{|l|}{ Chen 2012} \\
\hline \multicolumn{9}{|l|}{ Ho 2017} \\
\hline \multicolumn{9}{|l|}{ Ho 2019} \\
\hline \multicolumn{9}{|l|}{ Oudeman 2018} \\
\hline \multicolumn{9}{|l|}{ Su 2019} \\
\hline \multicolumn{9}{|c|}{ Tagliafico 2011} \\
\hline \multicolumn{9}{|l|}{ Vargas 2010} \\
\hline \multicolumn{9}{|l|}{ Wade 2020} \\
\hline \multicolumn{9}{|l|}{ Wade 2020} \\
\hline & $\begin{array}{l}\text { Domain } \\
\text { D1: Bias } \\
\text { D2: Bias } \\
\text { D3: Bias } \\
\text { D4: Bias } \\
\text { D5: Bias } \\
\text { D6: Bias } \\
\text { D7: Bias }\end{array}$ & $\begin{array}{l}\text { selecti } \\
\text { sificati } \\
\text { deviati } \\
\text { missin } \\
\text { surem } \\
\text { ction o }\end{array}$ & $\begin{array}{l}\text { rticipar } \\
\text { erventi } \\
\text { n inten } \\
\text { itcome } \\
\text { orted } r\end{array}$ & entio & & & & $\begin{array}{l}\text { ement } \\
\text { Serious } \\
\text { Moderate } \\
\text { Low } \\
\text { No information }\end{array}$ \\
\hline
\end{tabular}

Fig. 3 The summary risk of bias plot for included studies. Red = high risk, yellow $=$ unclear risk, green $=$ low risk

and clinicians interpret our summary values with both caution and respect to their particular circumstances.

In this study, the $N_{\mathrm{D}}$ was strongly related to the FA whereby fewer directions were associated with higher estimates of the FA. This is a well-known phenomenon [41] and likely to be explained by the association between noise (which can couple to give rise to anisotropy) and artefactually high estimates of the FA [37]. Whilst the observed FA values in the studies with relatively fewer directions and lower SNR appear plausible (rather than e.g. 0.9 which would clearly be artefactual), it is possible that such studies are more susceptible to bias and their estimates of FA are falsely high. Equally, our model may still be subject to collinearity because studies with higher $N_{\mathrm{D}}$ tended to also have a higher SNR. Conversely, as MD measures the size of the diffusion ellipsoid [45], we expected [41] MD to be independent of the $\mathrm{N}_{\mathrm{D}}$ and this is what we observed.

As humans age, axons lose their integrity, peripheral nerves demyelinate and there is a corresponding increase in extra-cellular fluid. Consequently, advancing aging is typically associated with reduced FA and increased diffusivity in white matter structures $[31,46]$. Prior work by Kronlage et al. [47] on the peripheral nerves in the forearm showed that the FA reduced with age (as in the brain [31]). We observed no statistically significant association between age and the FA of the roots of the brachial plexus but this may be due to the narrow age range of participants in our study. Whilst Kronlage [47] found that MD increased with age, we found that MD was slowed by $0.03 \times 10^{-3} \mathrm{~mm}^{2} / \mathrm{s}$ with each year of life (Fig. 5). Our findings are in agreement with the wider literature on age-related diffusivity changes in the brain [46] and compatible with the biological mechanisms of aging. Specifically, we observed that the MD of the roots of the brachial plexus slowed in the 3rd and 4th decade of life, which has also been observed in the healthy senescent adult brain [46]. It is unclear why Kronlage's work differs but this might be due to discrepancies in the age range of the sample (adults in this review were aged $28-45$ years versus 20-80 years in Kronlage's [47]) and aspects of the scanning because Kronlage [47] used non-isotropic voxels $(4.0 \mathrm{~mm}$ through plane, $1.5 \mathrm{~mm}^{2}$ in plane) which might underestimate the FA and overestimate the MD [43].

\section{Limitations}

The main limitation of this review is the pooled estimation of FA and MD. We decided to perform meta-analyses in the presence of high statistical heterogeneity because 
Table 2 Mixed-effects generalised linear modeling showing the unadjusted, multivariable and bootstrapped multivariable effect estimates of covariables on fractional anisotropy and mean diffusivity of the roots of the brachial plexus

\begin{tabular}{|c|c|c|c|c|c|c|c|}
\hline DTI parameter & Experimental factors & $\begin{array}{l}\text { Unadjusted coeffi- } \\
\text { cients }(95 \% \mathrm{CI})\end{array}$ & $p$ value & $\begin{array}{l}\text { Adjusted coefficients } \\
(95 \% \mathrm{CI})\end{array}$ & $p$ value & $\begin{array}{l}\text { Resampled adjusted } \\
\text { coefficients }(95 \% \mathrm{CI})\end{array}$ & $\begin{array}{l}\text { Resa- } \\
\text { mpled } \\
p \\
\text { value }\end{array}$ \\
\hline \multirow[t]{6}{*}{ Fractional anisotropy } & SNR & $\begin{array}{l}0.0001(-0.0003, \\
0.0004)\end{array}$ & 0.662 & $\begin{array}{l}0.0001(-0.0001, \\
0.0004)\end{array}$ & 0.257 & $\begin{array}{l}0.0001(-0.001 \\
0.002)\end{array}$ & 0.840 \\
\hline & Age in years & $\begin{array}{l}0.0003(-0.006 \\
0007)\end{array}$ & 0.926 & $0.001(0.001,0.002)$ & 0.001 & $\begin{array}{l}0.001(-0001 \\
0.003)\end{array}$ & 0.236 \\
\hline & $\begin{array}{l}\text { Number of diffusion } \\
\text { directions }\end{array}$ & $\begin{array}{l}-0.002(-0.005 \\
0001)\end{array}$ & 0.183 & $\begin{array}{l}-0.001(-0.002 \\
-0.001)\end{array}$ & $<0.001$ & $\begin{array}{l}-0.001(-0.002 \\
-0.0004)\end{array}$ & 0.002 \\
\hline & Echo time (ms) & $\begin{array}{l}0.002[0.00001, \\
0.004])\end{array}$ & 0.174 & $\mathrm{a}$ & $\mathrm{a}$ & $\mathrm{a}$ & $\mathrm{a}$ \\
\hline & $b$ value $(\mathrm{mm} / \mathrm{s})$ & $\begin{array}{l}-0.0004(-0.001 \\
0.0001)\end{array}$ & 0.098 & $\mathrm{a}$ & $\mathrm{a}$ & $\mathrm{a}$ & $\mathrm{a}$ \\
\hline & Resolution $\left(\mathrm{mm}^{3}\right)$ & $0.008(0.003,0.013)$ & 0.126 & a & a & $\mathrm{a}$ & $\mathrm{a}$ \\
\hline \multirow[t]{6}{*}{$\begin{array}{l}\text { Mean diffusivity } \\
\left(\mathrm{mm}^{2} / \mathrm{s} \times 10^{-3}\right)\end{array}$} & SNR & $\begin{array}{l}-0.001(-0.003 \\
0.0005)\end{array}$ & 0.154 & $-0.001(-0.003,0)$ & 0.055 & $\begin{array}{l}-0.001(-0.012 \\
0.01)\end{array}$ & 0.799 \\
\hline & Age in years & $-0.01(-0.03,0.01)$ & 0.303 & $\begin{array}{l}-0.025(-0.030 \\
-0.021)\end{array}$ & $<0.001$ & $\begin{array}{l}-0.03(-0.042 \\
-0.01)\end{array}$ & 0.003 \\
\hline & $\begin{array}{l}\text { Number of diffusion } \\
\text { directions }\end{array}$ & $0.005(-0003,0013)$ & 0.234 & $\begin{array}{l}0.001(-0.001 \\
0.002)\end{array}$ & 0.238 & $\begin{array}{l}0.001(-0.005 \\
0.006)\end{array}$ & 0.723 \\
\hline & Echo time (ms) & $\begin{array}{l}-0.012(-0.019 \\
-0.005)\end{array}$ & 0.174 & a & $\mathrm{a}$ & a & a \\
\hline & $b$ value $(\mathrm{mm} / \mathrm{s})$ & $0.001(0.0004,0.002)$ & 0.003 & a & a & a & a \\
\hline & Resolution $\left(\mathrm{mm}^{3}\right)$ & $0.004(0.002,0.007)$ & 0.562 & a & a & a & a \\
\hline
\end{tabular}

${ }^{a}$ Excluded due to multicollinearity

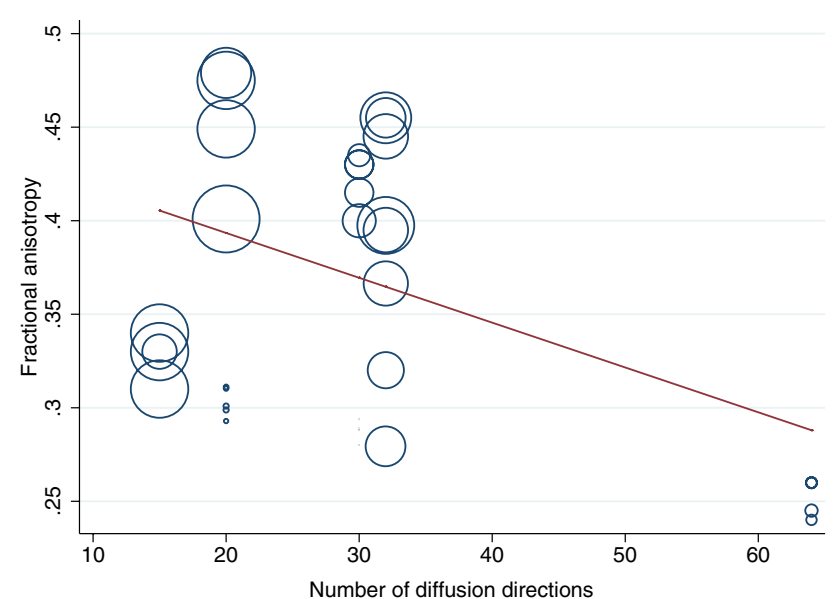

Fig. 4 A scatterplot showing the negative association between the mean fractional anisotropy of the roots of the brachial plexus and the number of diffusion sensitising gradient directions

(a) the generated outputs provide an important graphical representation of the variability of measurements in relation to experimental conditions which are easy to interpret, and (b) the forest plots provide a pictorial representation of the deleterious effects of failing to adjust study-level estimates for repeated measures. Figures 6 and

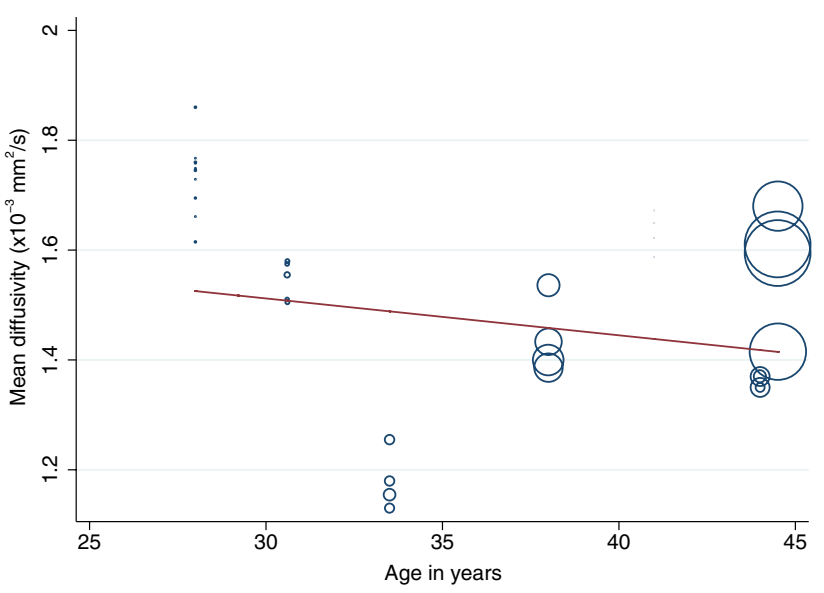

Fig. 5 A scatterplot showing the negative association between the mean diffusivity of the roots of the brachial plexus and the mean age of adults in the included studies

7 and Supplementary Figs. 2 and 3 show that in the eight studies [18, 21-24, 26, 28, 29] which did not use multilevel models, the standard errors of the mean (and thus, their CIs) are falsely small. We believe that if studies had appropriately adjusted for clustering/repeated measures, 




Fig. 6 Summary estimates of the normal fractional anisotropy of the roots of the brachial plexus at 3-T

the CIs would be wider, so overlaping the aggregate means from other studies and the measures of statistical heterogeneity (e.g. $I^{2}$ ) would fall.
We show a negative association between MD and age; however, readers should note that the range of aggregate ages in the included studies is narrow (28-45 years) and so the estimates may not be generalisable to the population. 


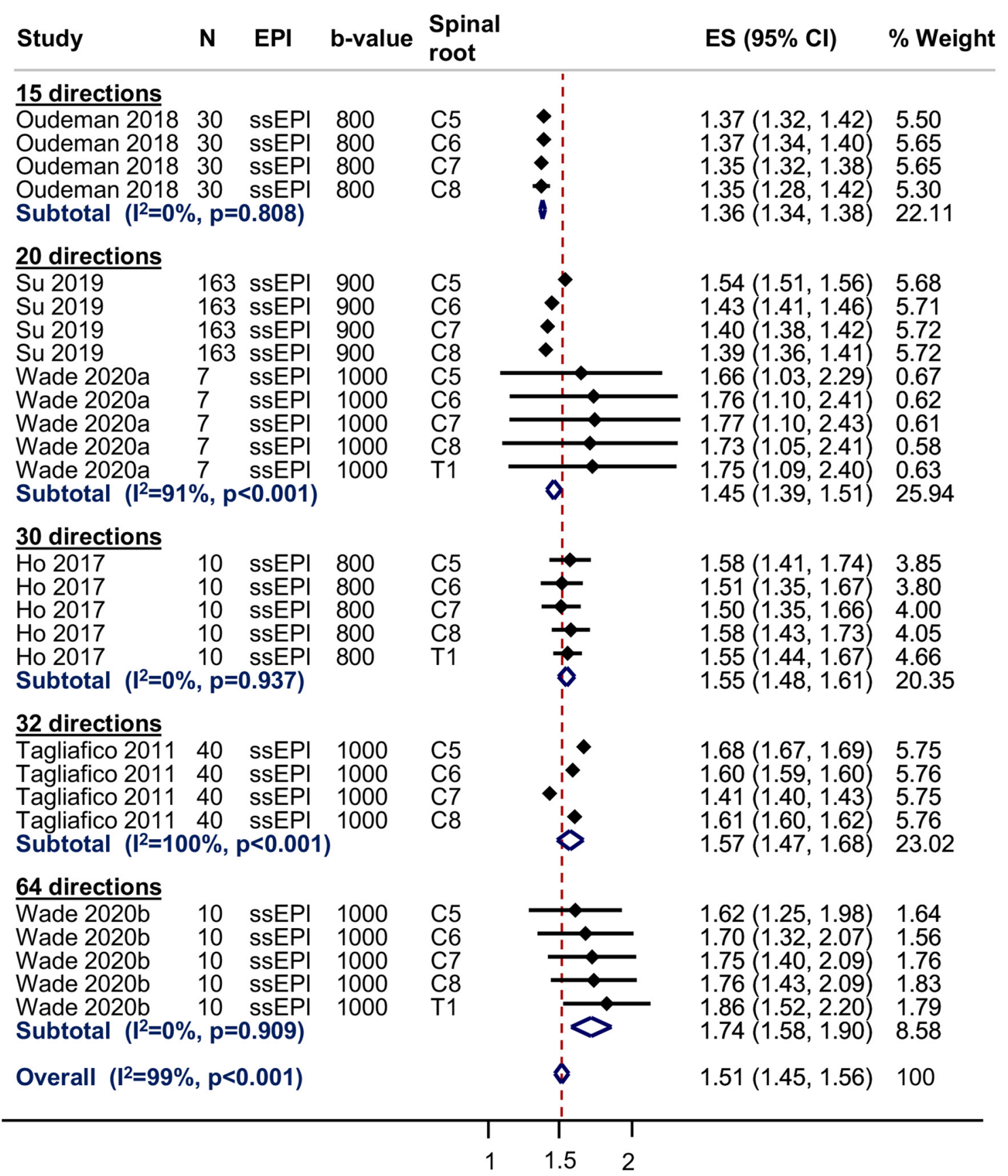

Fig. 7 Summary estimates of the normal mean diffusivity of the roots of the brachial plexus at 3-T

\section{Conclusions}

The roots of the brachial plexus in adults appear to have a pooled mean fractional anisotropy of $0.36(95 \% \mathrm{CI}$
$0.34,0.38)$ and pooled mean diffusivity of $1.51 \times 10^{-3}$ $\mathrm{mm}^{2} / \mathrm{s}(95 \%$ CI $1.45,1.56)$, although these parameters are dependent on experimental conditions and vary slightly from C5 to T1. 




Fig. 8 A funnel plot showing no evidence of small-study effects

Acknowledgements We thank Yuan Chi, Researcher at the Institute for Complementary and Integrative Medicine, University Hospital Zurich, Switzerland for translating an included article from Chinese to English and Helena Roess for translating an article we screened from Czech to English, which was facilitated through Cochrane's TaskExchange platform (https://community.cochrane.org/help/tools-and-software/ taskexchange). We also wish to thank Yemisi Takwoingi DVM MSc $\mathrm{PhD}$, Senior Research Fellow in Biostatistics in the Test Evaluation Research Group within the Institute of Applied Health Research, University of Birmingham for her advice on the statistical approach. We thank Donald Sammut FRCS FRCS(Plast) for generously permitting the reproduction of his artwork in this manuscript (https://www.donal dsammut.com).

Author contributions RGW conceived and designed the study, registered the protocol, extracted and analysed data, performed risk of bias assessments and co-authored the manuscript. AW extracted data, performed risk of bias assessments and co-authored the manuscript. IT, GA, FY and MW supervised the analysis of data analysis, interpretation and co-authored the manuscript. GB had oversight of the entire project, supervising the design, analysis and interpretations, and coauthored the manuscript.

Funding Ryckie Wade is a Doctoral Research Fellow funded by the National Institute for Health Research (NIHR, DRF-2018-11-ST2-028). This research is also supported by the NIHR Leeds Biomedical Research Centre and University of Leeds Advanced Imaging Centre, which is funded by the Medical Research Council with support from the British Heart Foundation and Arthritis Research UK. Funding was also given by the Wallenberg Centre for Molecular Medicine and the Västerbotten County Council, Sweden. The views expressed are those of the author(s) and not necessarily those of the United Kingdom's National Health Service, NIHR or Department of Health.

Availability of data The extracted data and statistical syntax are available from the first author upon request.

\section{Compliance with ethical standards}

Conflict of interest None.
Open Access This article is licensed under a Creative Commons Attribution 4.0 International License, which permits use, sharing, adaptation, distribution and reproduction in any medium or format, as long as you give appropriate credit to the original author(s) and the source, provide a link to the Creative Commons licence, and indicate if changes were made. The images or other third party material in this article are included in the article's Creative Commons licence, unless indicated otherwise in a credit line to the material. If material is not included in the article's Creative Commons licence and your intended use is not permitted by statutory regulation or exceeds the permitted use, you will need to obtain permission directly from the copyright holder. To view a copy of this licence, visit http://creativecommons.org/licenses/by/4.0/.

\section{References}

1. Vargas MI, Viallon M, Nguyen D, Beaulieu JY, Delavelle J, Becker M (2010) New approaches in imaging of the brachial plexus. Eur J Radiol 74:403-410

2. Nardin RA, Patel MR, Gudas TF, Rutkove SB, Raynor EM (1999) Electromyography and magnetic resonance imaging in the evaluation of radiculopathy. Muscle Nerve 22:151-155

3. Zhu Y-S, Mu N-N, Zheng M-J, Zhang Y-C, Feng H, Cong R et al (2014) High-resolution ultrasonography for the diagnosis of brachial plexus root lesions. Ultrasound Med Biol 40:1420-1426

4. Mallouhi A, Meirer R, Bodner G (2003) Sonographic features of brachial plexus traumatic rupture. J Neurosurg 99:432-432

5. Lapegue F, Faruch-Bilfeld M, Demondion X, Apredoaei C, Bayol MA, Artico H et al (2014) Ultrasonography of the brachial plexus, normal appearance and practical applications. Diagn Interv Imaging 95:259-275

6. Sureka J, Cherian RA, Alexander M, Thomas BP (2009) MRI of brachial plexopathies. Clin Radiol 64:208-218

7. Wade RG, Takwoingi Y, Wormald JCR, Ridgway JP, Tanner S, Rankine JJ et al (2019) MRI for detecting root avulsions in traumatic adult brachial plexus injuries: a systematic review and meta-analysis of diagnostic accuracy. Radiology 293:125-133

8. Chin B, Ramji M, Farrokhyar F, Bain JR (2017) Efficient imaging: examining the value of ultrasound in the diagnosis of traumatic adult brachial plexus injuries, a systematic review. Neurosurgery 83:323-332. https://doi.org/10.1093/neuros/nyx483

9. Van der Linde E, Naidu V, Mitha A, Rocher A (2015) Diagnosis of nerve root avulsion injuries in adults with traumatic brachial plexopathies: MRI compared with CT myelography. S Afr J Radiol 19:1-9

10. Nath V, Schilling KG, Parvathaneni P, Huo Y, Blaber JA, Hainline AE et al (2020) Tractography reproducibility challenge with empirical data (TraCED): the 2017 ISMRM diffusion study group challenge. J Magn Reson Imaging 51:234-249

11. Vavasour IM, Meyers SM, Mädler B, Harris T, Fu E, Li DKB et al (2019) Multicenter measurements of T1 relaxation and diffusion tensor imaging: intra and intersite reproducibility. J Neuroimaging 29:42-51

12. Prohl AK, Scherrer B, Tomas-Fernandez X, Filip-Dhima R, Kapur K, Velasco-Annis C et al (2019) Reproducibility of structural and diffusion tensor imaging in the TACERN multi-center study. Front Integr Neurosci 13:1-15

13. Kimura M, Yabuuchi H, Matsumoto R, Kobayashi K, Yamashita Y, Nagatomo K et al (2020) The reproducibility of measurements using a standardization phantom for the evaluation of fractional anisotropy (FA) derived from diffusion tensor imaging (DTI). Magn Reson Mater Physics, Biol Med. 33(2):293-298 
14. Heckel A, Weiler M, Xia A, Ruetters M, Pham M, Bendszus M et al (2015) Peripheral nerve diffusion tensor imaging: assessment of axon and myelin sheath integrity. PLoS ONE 10:e0130833

15. Andersson G, Orädd G, Sultan F, Novikov LN (2018) In vivo diffusion tensor imaging, diffusion kurtosis imaging, and tractography of a sciatic nerve injury model in rat at $9.4 \mathrm{~T}$. Sci Rep 8:12911

16. Higgins JPT, Green S (eds) (2011) Cochrane Handbook for Systematic Reviews of Interventions Version 5.1.0 [updated March 2011]. Cochrane Collab

17. Moher D, Liberati A, Tetzlaff J, Altman DG (2009) Systematic reviews and meta-analyses: the PRISMA statement. Annu Intern Med 151:264-269

18. Wade RG, Tanner SF, Teh I, Ridgway JP, Shelley D, Chaka B et al (2020) Diffusion tensor imaging for diagnosing root avulsions in traumatic adult brachial plexus injuries: a proof-of-concept study. Front Surg 7:19. https://doi.org/10.3389/fsurg.2020.00019

19. Sterne JA, Hernán MA, Reeves BC, Savović J, Berkman ND, Viswanathan M et al (2016) ROBINS-I: a tool for assessing risk of bias in non-randomised studies of interventions. BMJ 355:i4919

20. McGuinness LA, Higgins JPT (2020) Risk-of-bias VISualization (robvis): an R package and Shiny web app for visualizing riskof-bias assessments. Res Synth Methods. https://doi.org/10.1002/ jrsm.1411 (in press)

21. Ho MJ, Ciritsis A, Manoliu A, Stieltjes B, Marcon M, Andreisek $G$ et al (2019) Diffusion tensor imaging of the brachial plexus: a comparison between readout-segmented and conventional singleshot echo-planar imaging. Magn Reson Med Sci 18:150-157

22. Ho MJ, Manoliu A, Kuhn FP, Stieltjes B, Klarhöfer M, Feiweier $T$ et al (2017) Evaluation of reproducibility of diffusion tensor imaging in the brachial plexus at 3.0 T. Invest Radiol. 52:482-487

23. Chen M, Li X, Chen J, Sun C, He J (2012) Quantitative analysis of the normal brachial plexus with diffusion tensor imaging. Chin J Med Imaging Technol 28:77-81

24. Oudeman J, Verhamme C, Engbersen MP, Caan MWA, Maas M, Froeling $\mathrm{M}$ et al (2018) Diffusion tensor MRI of the healthy brachial plexus. PLoS ONE 13:e0196975

25. Su X, Kong X, Liu D, Kong X, Alwalid O, Wang J et al (2019) Multimodal magnetic resonance imaging of peripheral nerves: establishment and validation of brachial and lumbosacral plexi measurements in 163 healthy subjects. Eur J Radiol 117:41-48

26. Vargas MI, Viallon M, Nguyen D, Delavelle J, Becker M (2010) Diffusion tensor imaging (DTI) and tractography of the brachial plexus: feasibility and initial experience in neoplastic conditions. Neuroradiology 52:237-245

27. Wade RG, Teh I, Andersson G, Fang-Cheng Y, Wiberg M, Bourke $\mathrm{G}$ (2020) Diffusion tensor imaging of the brachial plexus: defining the fractional anisotropy threshold for deterministic tractography of the roots. FigShare Prepr. https://doi.org/10.6084/m9.figsh are. 12173184

28. Tagliafico A, Calabrese M, Puntoni M, Pace D, Baio G, Neumaier CE et al (2011) Brachial plexus MR imaging: accuracy and reproducibility of DTI-derived measurements and fibre tractography at 3.0-T. Eur Radiol 21:1764-1771

29. Samardzic M, Rasulic L, Grujicic D, Bacetic D, Milicic B (2011) Nerve transfers using collateral branches of the brachial plexus as donors in patients with upper palsy-thirty years' experience. Acta Neurochir (Wien) 153:2009-2019

30. Lebel C, Gee M, Camicioli R, Wieler M, Martin W, Beaulieu C (2012) Diffusion tensor imaging of white matter tract evolution over the lifespan. Neuroimage 60:340-352

31. Yap QJ, Teh I, Fusar-Poli P, Sum MY, Kuswanto C, Sim K (2013) Tracking cerebral white matter changes across the lifespan: insights from diffusion tensor imaging studies. J Neural Transm 120:1369-1395
32. Gesslbauer B, Hruby LA, Roche AD, Farina D, Blumer R, Aszmann OC (2017) Axonal components of nerves innervating the human arm. Ann Neurol 82:396-408

33. Aboitiz F, Scheibel AB, Fisher RS, Zaidel E (1992) Fiber composition of the human corpus callosum. Brain Res 598:143-153

34. Yokoyama I (1989) Study on the intraneural topography of the brachial plexus. Nihon Seikeigeka Gakkai Zasshi Japan 63:1085-1102

35. Sinha S, Prasad GL, Lalwani S (2016) A cadaveric microanatomical study of the fascicular topography of the brachial plexus. J Neurosurg 125:355-362

36. Helmer KG, Chou M-C, Preciado RI, Gimi B, Rollins NK, Song A et al (2016) Multi-site study of diffusion metric variability: effects of site, vendor, field strength, and echo time on regions-of-interest and histogram-bin analyses. In: Gimi B, Krol A (eds) Physiol Behav, p $97882 \mathrm{U}$

37. Jones DK, Basser PJ (2004) "Squashing peanuts and smashing pumpkins": how noise distorts diffusion-weighted MR data. Magn Reson Med 52:979-993

38. Schilling KG, Nath V, Blaber J, Harrigan RL, Ding Z, Anderson AW et al (2017) Effects of b-value and number of gradient directions on diffusion MRI measures obtained with Q-ball imaging. In: Styner MA, Angelini ED (eds) Proc SPIE Int Soc Opt Eng., $p$ $101330 \mathrm{~N}$

39. Qin W, Shui YuC, Zhang F, Du XY, Jiang H, Xia Yan Y et al (2009) Effects of echo time on diffusion quantification of brain white matter at 15T and 30T. Magn Reson Med 61:755-760

40. Haakma W, Pedersen M, Froeling M, Uhrenholt L, Leemans A, Boel LWT (2016) Diffusion tensor imaging of peripheral nerves in non-fixed post-mortem subjects. Forensic Sci Int 263:139-146

41. Giannelli M, Cosottini M, Michelassi MC, Lazzarotti G, Belmonte G, Bartolozzi C et al (2010) Dependence of brain DTI maps of fractional anisotropy and mean diffusivity on the number of diffusion weighting directions. J Appl Clin Med Phys 11:176-190

42. Taylor PA, Alhamud A, van der Kouwe A, Saleh MG, Laughton B, Meintjes E (2016) Assessing the performance of different DTI motion correction strategies in the presence of EPI distortion correction. Hum Brain Mapp 37:4405-4424

43. Haddad SMH, Scott CJM, Ozzoude M, Holmes MF, Arnott SR, Nanayakkara ND et al (2019) Comparison of quality control methods for automated diffusion tensor imaging analysis pipelines. PLoS ONE 14:e0226715

44. Vos SB, Jones DK, Viergever MA, Leemans A (2011) Partial volume effect as a hidden covariate in DTI analyses. Neuroimage 55:1566-1576

45. Le Bihan D, Mangin J-F, Poupon C, Clark CA, Pappata S, Molko $\mathrm{N}$ et al (2001) Diffusion tensor imaging: concepts and applications. J Magn Reson Imaging 13:534-546

46. Watanabe M, Sakai O, Ozonoff A, Kussman S, Jara H (2013) Age-related apparent diffusion coefficient changes in the normal brain. Radiology 266:575-582

47. Kronlage M, Schwehr V, Schwarz D, Godel T, Uhlmann L, Heiland $S$ et al (2018) Peripheral nerve diffusion tensor imaging (DTI): normal values and demographic determinants in a cohort of 60 healthy individuals. Eur Radiol 28:1801-1808

Publisher's Note Springer Nature remains neutral with regard to jurisdictional claims in published maps and institutional affiliations. 